\title{
A Simple Program for Fast Tilting Electron-Beam Sensitive Crystals to Zone Axes
}

Yanhang $\mathrm{Ma}^{1}$ and $\mathrm{Tu} \mathrm{Sun}^{2}$

${ }^{1}$ ShanghaiTech University, Shanghai, China (People's Republic), ${ }^{2}$ ShanghaiTech University, United States

Tilting crystals to proper zone axes is a necessary but tedious work in taking selected area electron diffraction patterns (SAED) and high-resolution images using transmission electron microscope (TEM). This process not only costs a lot of time but also limits the application of TEM in electron-beam sensitive materials. In most cases, the alignment of crystals is usually done manually by trial and error, which heavily rely on the experiences of the operator [1]. Therefore, it is desirable to develop a simple method for tilting crystals from random orientations to a specific zone axis quickly. Such a tool will prompt the study of electron-beam sensitive materials, such as zeolites and metal-organic frameworks (MOFs), as their structures cannot sustain a long-time exposure [2-4]. Kikuchi patterns under convergent-beam electron diffraction (CBED) mode have been widely used for accurately determining orientations of materials [5-8], while the high density of electrons will destroy the materials quickly. A tool "KSpaceNavigator" [9] has been developed for sample tilting in high-resolution imaging, tomography and defect analysis. Also, a method has been reported recently [10] of tilting MOFs to zone axes with assistance of computer program while it requires the range of misorientation less than $\pm 5^{\circ}$. Herein, we describe a novel program, Zones, which can index the electron diffraction pattern and calculate the tilting angles of a double-tilt sample holder from the current orientation to a desired zone axis. It can also bring crystals that are slightly deviated from a zone axis to the exact zone with the help of Laue ring in the diffraction pattern. This program has been successfully applied to studies of zeolites and metal-organic frameworks (MOFs), known as being electron-beam sensitive. The program shows its power not only in saving the operator's time but also in preventing the crystals from quick beam damages.

\section{Acknowledgement:}

The work was supported by the Shanghai Pujiang Program (17PJ1406400), the Commission for Science and Technology of Shanghai Municipality (17ZR1418600), the Young Elite Scientist Sponsorship Program by CAST (2017QNRC001), the National Natural Science Foundation of China (21875140), the National Key Research and Development Program of China (21835002) and CћEM, SPST of ShanghaiTech University(\#EM02161943). We thank Prof. Osamu Terasaki and Dr. Peter Oleynikov for helpful discussions. 
a

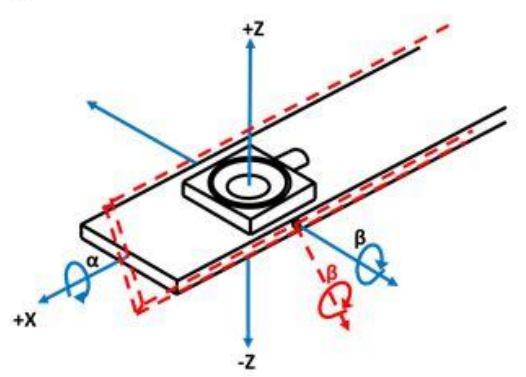

d

Initial orientation

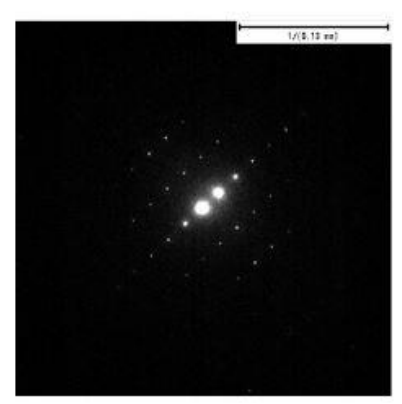

b

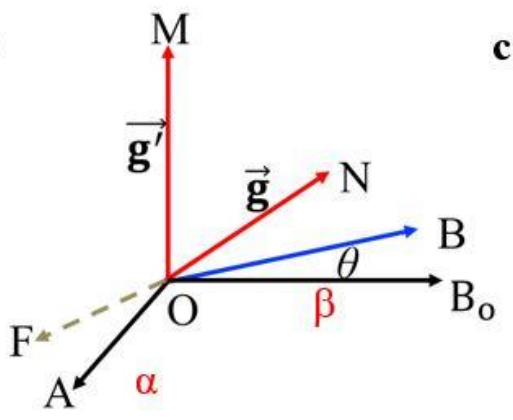

e

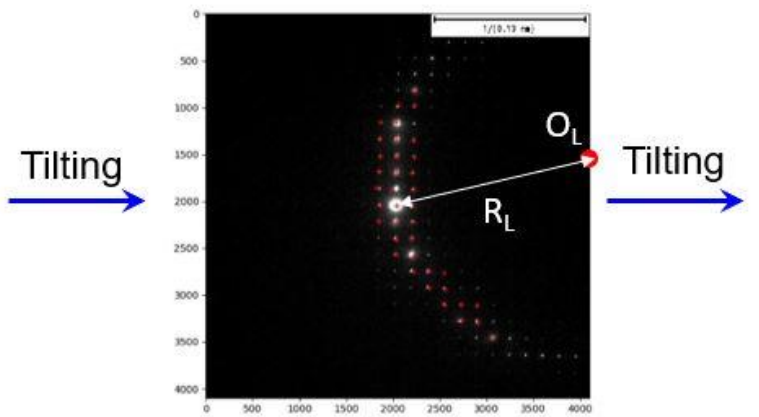

c

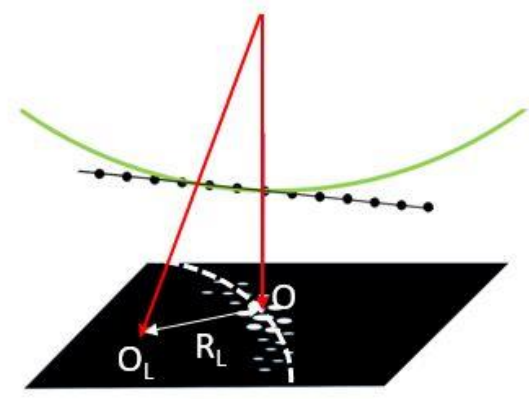

Targeted zone axis

Figure 1. Figure 1. (a-c) Illustration of crystal tilting from one direction to another, (d-e) experimental data.

\section{References}

[1] D.B. Williams, C.B. Carter, Transmission Electron Microscopy: A Textbook For Materials Science, second ed., Springer, 2009.

[2] C. Wiktor, M. Meledina, S. Turner, O.I. Lebedev, R.A. Fischer, M.M.J. Treacy, J. Newsam. Ultramicroscopy 23 (1987) 411-419.

[3] C. Wiktor, M. Meledina, S. Turner, O.I. Lebedevd, R.A. Fischere. J. Mater. Chem. A 5 (2017) 1496914989.

[4] O. Terasaki, J.M. Thomas, G.R. Millward. Proc. R. Soc. Lond. A 395 (1984) 153-164.

[5] M. von Heimendahl, W. Bell, G. Thomas, J. Appl. Phys. 35 (1964) 3614-3616.

[6] R.C. Ecob, M.P. Shaw, A.J. Porter, B. Ralph. Philos. Mag. A 44 (1981)1117-1133.

[7] P. Heilmann, W.A.T. Clark, D.A. Rigney. Ultramicroscopy 9 (1982) 365-371.

[8] Q. Liu. Ultramicroscopy 60 (1995) 81-89.

[9] T. Duden, A. Gautam, U. Dahmen. Ultramicroscopy 111 (2011) 1574-1580.

[10] D.L. Zhang, Y.H. Zhu, L.M. Liu, X.R. Ying, C. Hsiung, R. Sougrat, K. Li, Y. Han. Science 359 (2018) 675-679, 